\title{
Addendum: Cryptic phosphorylation in nucleoside natural product biosynthesis
}

Matthew M. Draelos, Anyarat Thanapipatsiri (D), Hilda Sucipto and Kenichi Yokoyama (D)

Addendum to: Nature Chemical Biology https://doi.org/10.1038/s41589-020-00656-8, published online 30 November 2020

After publication, we identified errors in the stereochemical assignments of two compounds in the original published work and, through this addendum, correct these assignments. Upon further characterization studies of $5^{\prime}$-amino- $6^{\prime}$-hydroxy-octosyl acid (AHOA), we are revising its stereochemistry, and that of $\operatorname{AHOAP}(\mathbf{1 6})$, at the $4^{\prime}, 5^{\prime}, 6^{\prime}$, and $7^{\prime}$ positions (Supplementary Fig. 17a,b). In our recent characterization of AHOA by two-dimensional Nuclear Overhauser Spectroscopy (NOESY) experiments, the data revealed a strong correlation between proton signals at $\delta 4.42$ and $3.95 \mathrm{ppm}$ (red dashed circle in Supplementary Fig. 17c). Originally, these two signals were assigned as $\mathrm{H} 2^{\prime}$ and $\mathrm{H}^{\prime}$, respectively. However, they are positioned on opposite sides of the molecule and too spatially separated ( 5.9 $\left.\AA\right)$ to yield a strong NOESY correlation. Consequently, we revisited the original ${ }^{1} \mathrm{H}$ NMR assignment and noticed an alternative interpretation of the original ${ }^{1} \mathrm{H}-{ }^{1} \mathrm{H}$ COSY data (compare Supplementary Fig. 17e,f). This alternative assignment was also consistent with the newly determined HMBC spectrum of AHOA (Supplementary Fig. 17g), in which the observed $\mathrm{H}^{\prime}{ }^{\prime}-\mathrm{C} 2{ }^{\prime}$ and $\mathrm{H}^{\prime}$ '-C8' correlations are consistent with the alternative assignment and inconsistent with the original assignment. With this alternative assignment, analysis of $J$-coupling constants suggested a revised stereochemistry of AHOA (Supplementary Figure 17b). This structure is fully consistent with the NOESY data (Supplementary Fig. 17d), and all the NOESY correlations are between protons with distances within $2.6 \AA$. Furthermore, the revised structure eliminates the requirement for the epimerization originally proposed to occur during the transformation of HKOAP to AHAP via AHOAP. The structures of AHOA and AHOAP in Figs. 1, 3, and 5, Extended Data Fig. 10, Supplementary Fig. 9 and the Supplementary Note and the corresponding text have now been corrected. Supplementary Fig. 17 has been added to the Supplementary Information, and the new NOESY and HMBC spectra have also been added to the Supplementary Note. 

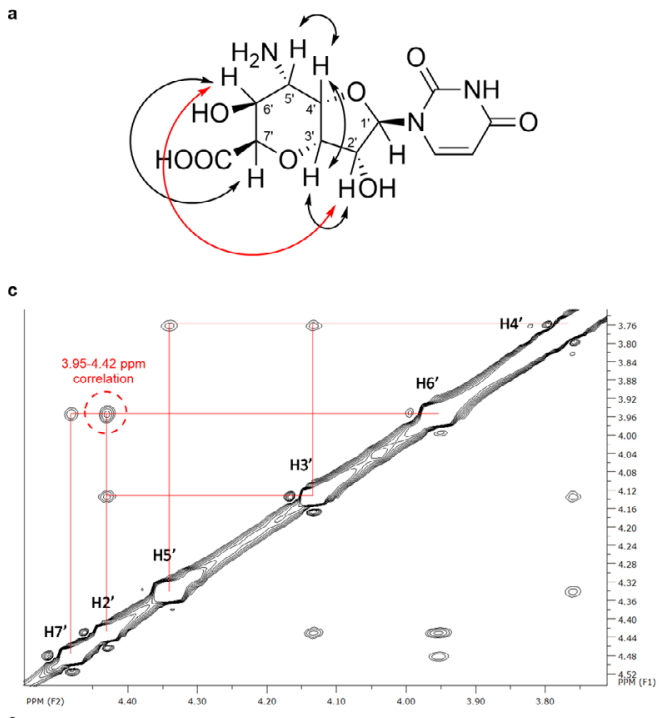
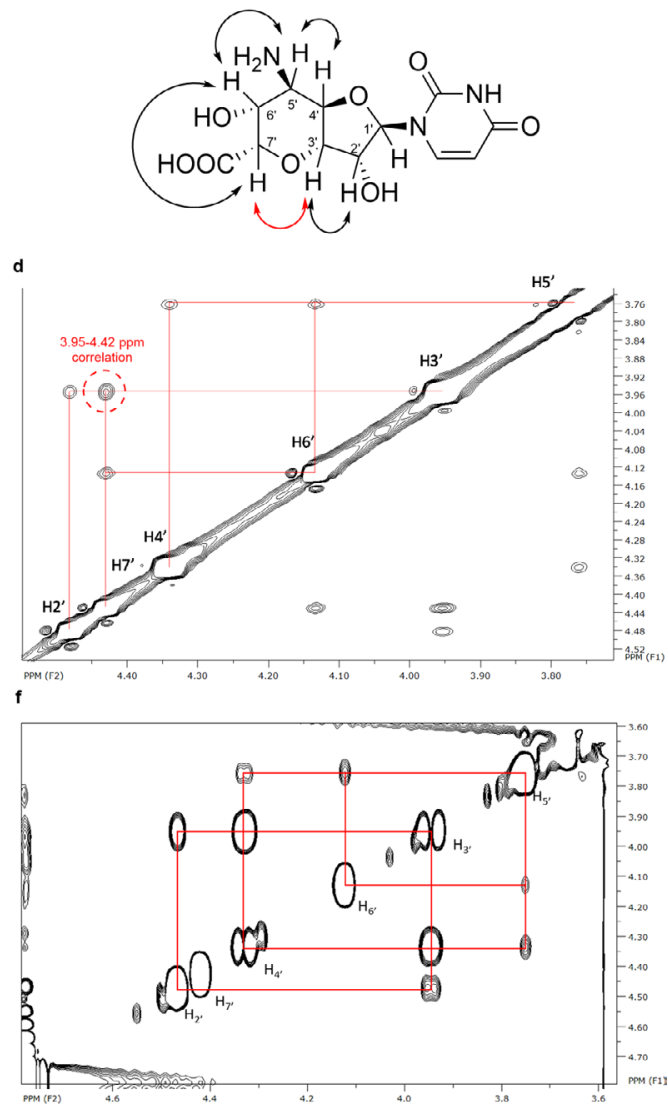

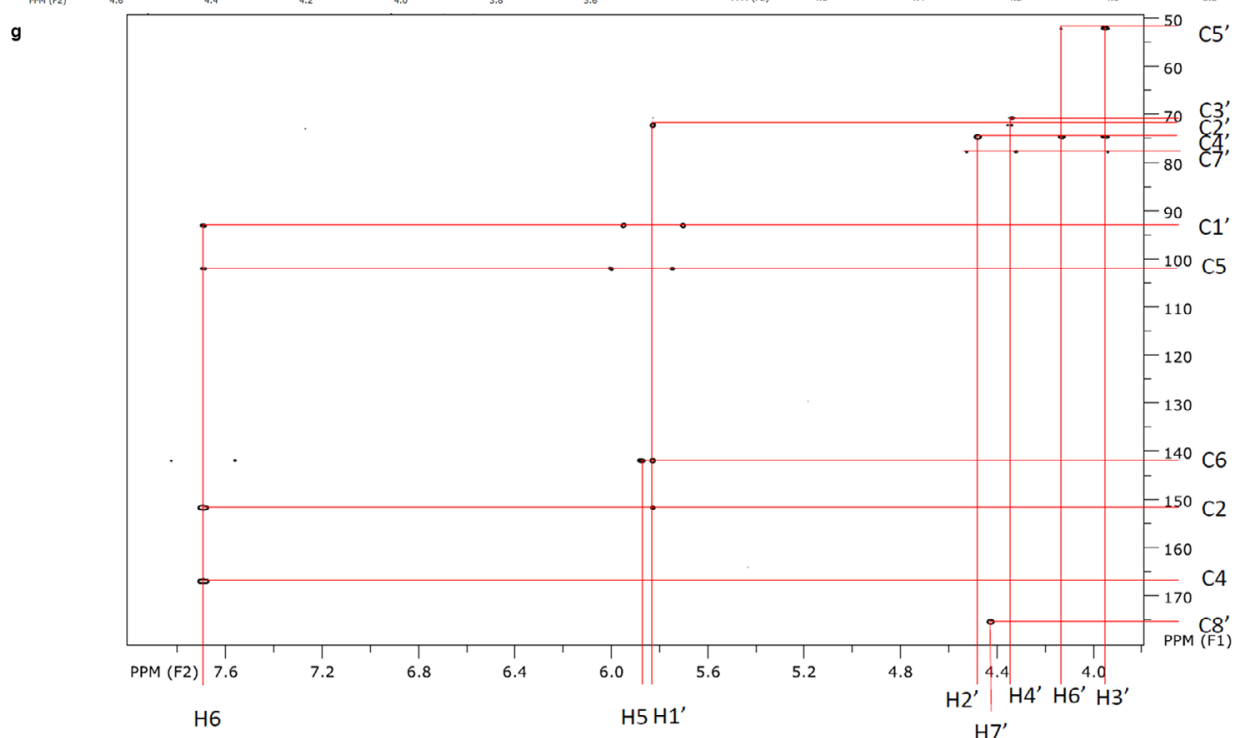

Supplementary Figure 17. Stereochemical revision of AHOA. a and b. Original (a) and revised (b) structure of AHOA with NOESY correlations shown in double-headed arrows (3.95 -4.42 ppm correlation shown in red). $\mathbf{c}$ and $\mathbf{d}$. ${ }^{1} \mathrm{H}-{ }^{1} \mathrm{H}$ NOESY spectrum of AHOA with the original (c) and revised (d) signal assignment. e and $\mathbf{f} .{ }^{1} \mathrm{H}-{ }^{-1} \mathrm{H}$ COSY spectrum of $\mathrm{AHOA}$ with original (e) and revised (f) signal assignments. $\mathbf{g}$. $\mathrm{HMBC}$ spectrum of $\mathrm{AHOA}$ with the revised signal assignments.

Published online: 11 August 2021

https://doi.org/10.1038/s41589-021-00867-7

(c) The Author(s), under exclusive licence to Springer Nature America, Inc. 2021 